Article

\title{
On the High-Energy Neutrino Emission from Active Galactic Nuclei ${ }^{\dagger}$
}

\author{
Emma Kun ${ }^{1, *(1)}$, Peter L. Biermann ${ }^{2,3,4,5}$, Silke Britzen ${ }^{2}$ and László Á. Gergely ${ }^{1}$ (1) \\ 1 Institute of Physics, University of Szeged, Dóm tér 9, H-6720 Szeged, Hungary; gergely@physx.u-szeged.hu \\ 2 Max Planck Institute for Radioastronomy, Auf dem Hügel 69, D-53121 Bonn, Germany; \\ plbiermann@mpifr-bonn.mpg.de (P.L.B.); sbritzen@mpifr-bonn.mpg.de (S.B.) \\ 3 Department of Physics, Karlsruhe Institute for Technology, P.O. Box 3640, D-76021 Karlsruhe, Germany \\ 4 Department of Physics \& Astronomy, University of Alabama, Tuscaloosa, AL 35487-0324, USA \\ 5 Department of Physics \& Astronomy, University of Bonn, Regina-Pacis-Weg 3, 53113 Bonn, Germany \\ * Correspondence: kun@titan.physx.u-szeged.hu \\ + This paper is an extended version of our paper published in BGL 17: 10th Bolyai-Gauss-Lobachevsky \\ Conference on Non-Euclidean Geometry and its Applications, 20-26 August 2017, Gyöngyös, Hungary.
}

Received: 14 November 2017; Accepted: 17 January 2018; Published: 1 February 2018

\begin{abstract}
We review observational aspects of the active galactic nuclei and their jets in connection with the detection of high-energy neutrinos by the Antarctic IceCube Neutrino Observatory. We propose that a reoriented jet generated by the spin-flipping supermassive black hole in a binary merger is likely the source of such high-energy neutrinos. Hence they encode important information on the afterlife of coalescing supermassive black hole binaries. As the gravitational radiation emanating from them will be monitored by the future LISA space mission, high-energy neutrino detections could be considered a contributor to multi-messenger astronomy.
\end{abstract}

Keywords: supermassive black holes; gravitational waves; high-energy neutrino emission; active galactic nuclei; relativistic jets

\section{Introduction}

Electromagnetic radiation has been providing a first observational window to the Universe for about 400 years, while a second window opened with the detection of the first flavour of neutrinos in 1956 (electron neutrino, [1]) and the ultra-high energy cosmic ray particles (UHECRs) in 1962 [2]. The third observational window to the Universe opened up on 14th September 2015 [3], when the two Advanced LIGO observatories firstly detected a gravitational wave (GW) signal, emanated from the coalescence of two astrophysical black holes (BH). Up to date four GW detections together with a lower significance candidate from binary black hole sources, and a GW detection from colliding neutron stars were announced by the LIGO Scientific Collaboration and Virgo Collaboration [3-8].

High-frequency GWs (hfGW, of order of $\mathrm{Hz}$ to $\mathrm{kHz}$ ) are emitted from the coalescence of astrophysical BHs or neutron stars, while low-frequency GWs (lfGW, of order of microHz to milliHz) are emitted from the merging of supermassive black holes (SMBHs). The LIGO and Virgo detectors are sensitive to hfGWs. The direct detection of lfGWs is the purpose of the future Laser Interferometer Space Antenna (LISA) space mission [9], an experiment facilitating astrophysics and cosmology research in the $\mathrm{mHz}$ regime. Providing priors and predictions to this experiment is of paramount importance.

The compact centre of some galaxies is radiating tremendous energy across the electromagnetic spectrum due to the accretion onto the central SMBH, believed to be the main engine of the activity in active galactic nuclei (AGN; for a review see e.g., [10]). Observations on binary black hole candidates in kpc-scale separated binaries are naturally feasible with spatially resolved AGN. However, one can reveal 
the presence of a sub-pc-scale separated binary in AGN only by indirect approaches. When an AGN is radio-loud, its periodic jet structure might indicate, that the jet emitter black hole interacts gravitationally with another black hole (e.g., [11-17]). In absence of strong relativistic jets, the compact centre of the galaxy is observed as a radio-quiet AGN. In case of these AGN their emission in optical wavelengths might reveal the binary nature of the centre of the galaxy (e.g., [18]). Evidence for a merger is detectable for a long time period, starting just before the actual merger, and persisting for quite a long period afterwards, estimated in thousands of years, based on the traversal time scale for the jet to penetrate initially the inner high density region of the host galaxy (e.g., [19]).

\section{Active Galactic Nuclei and Their Jets}

According to the accepted evolutionary models, galaxies gather their masses through a sequence of accretion and merger phases (e.g., [20-22]). As a result of the process, million-to-billion solar mass SMBHs formed at the centre of the massive galaxies [23], with some of them members of binary systems due to the long time-scale of the merger [24,25]. An accretion disk may form around the $\mathrm{SMBH}$ due to the non-zero angular momentum of the infalling matter [26]. As substantial energy is released in the accretion disk due to its efficient mass-energy conversion [27], the compact centre of the galaxy becomes an AGN (e.g., [28]). SMBHs are believed to be the main engine of the activity in AGNs [10]. General relativistic modelling of magnetized jets from accreting BH showed, that provided the central spin is high enough [29], a pair of relativistic jets is launched from the immediate vicinity of the BH (e.g., [30]), composed mostly by electrons and positrons (in light jets), or electrons and protons (in heavy jets). These jets could be powered either by the rotational energy of the central SMBH (through the Blandford-Znajek process, [31]), or by the magnetized accretion disk wind accelerated by magneto-centrifugal forces (Blandford-Payne process, [32,33]).

Particles are accelerating on helical paths along the magnetic field lines, emitting synchrotron radiation (e.g., [34]). In the Fourier transform of the continuum spectrum of the synchrotron radiation the most powerful frequency is the critical frequency (e.g., [35]). The electron energies and magnetic field strengths typical to AGN render the critical frequency into the radio regime, so the collimated jets are observed as radio-loud AGN (approximately 10 per-cent of single AGN qualify [36,37]).

Apart from the synchrotron radiation in radio wavelengths, AGN and their jets emit energetic particles in the X-ray and $\gamma$-regime. According to the Pierre Auger and IceCube Collaborations the main sources of UHECRs and cosmic high-energy (HE) neutrinos are AGNs (e.g., [38-42]). Two interaction channels were proposed for neutrino production in AGN: one with protons interacting with ambient photons (from the accretion disk, synchrotron photons emitted from the jet, CMB photons strayed into the jet); and another with protons interacting with other protons within the jet or with protons of the external material trapped in the jet flow (e.g., [43-45]).

\section{High-Energy Neutrino Emission in AGN}

Various scenarios for HE neutrino production in AGN jets have been suggested (e.g., [46-49]); it is plausible to assume that the observed cosmic HE neutrino background originates from some combination of these. The common property of the models is that there has to be a process inducing shock-waves in the accretion disk or the jet, that accelerates protons to energies exceeding the threshold of pion-production, that decay further to HE neutrinos, among other particles. It means the incoming proton has to have at least a relativistic mass of 1.3 times its rest mass, and thus a kinetic energy around $290 \mathrm{MeV}$, or moving with at least 0.65 c (e.g., [50]). This condition is naturally satisfied in AGN jets, since the Lorentz factor of the turbulent flow is usually pretty high in such systems.

In [51] three of the present authors proposed a scenario of binary black hole evolution leading to the emission of HE neutrinos. The scenario relies on the final coalescence of two SMBHs, followed by the emission of lfGWs, an enormous shock-wave and the formation of a new energetic jet. The freshly started jet has high Lorentz factor, and is able to accelerate the particles leading to the emission of HE neutrinos. By cross-correlating both the Parkes Catalogue and the Second Planck Catalogue 
of Compact Sources with the arrival direction of the track-type neutrinos detected by the IceCube Neutrino Observatory, we identified four track events, all with flat spectrum radio sources, and of those again two with flat spectrum radio sources with their spectrum extending to near THz. The brightest of these two, the flat-spectrum blazar PKS 0723-008 emerged as a good candidate for the high-energy neutrino event 5 [51].

The following quantitative picture illustrates what may be common. We observe UHECR particles, and two scenarios are viable to explain them, with probably both realized in nature [52]: one is based on the relativistic jets and their shock waves in radio galaxies (e.g., [46]), and the other on Gamma Ray Bursts (GRBs) and their relativistic jets. Both sites of origin may involve mergers of black holes or neutron stars [51,53], or the ab initio formation of black holes. In a series of papers by Mannheim, Falcke and Markoff (e.g., [43,54,55]) it has been shown that at a distance of order $10^{3.5}$ to $10^{5}$ Schwarzschild radii from a central black hole a reconfinement shock system occurs, starting the routine jet flow (e.g., [56,57]). So when a magnetic jet system is newly established in a new direction [58] during a merger event, the jet flow has to be re-established (the characteristic velocity for this may be the relativistic speed of sound $c / \sqrt{3}$ ), particles have be newly injected, and built up as a viable population. The observation of a time lag between the GW event and the HE photons [59] of $1.74 \mathrm{~s}$ is compatible with this characteristic distance for a central black hole of a mass of order 1 to 2 Solar masses: the characteristic distance introduced above yields a range of expected time lags of $10^{-0.45}$ to $10^{+1.05} \mathrm{~s}$ for a neutron star merger resulting in a black hole, fully compatible with the observations; the additional time lags due to the build-up of a viable population of energetic particles may be as fast or even faster. This time scales with the mass of the central black hole, and so the merger of two super-massive black holes of order $10^{8}$ Solar masses the corresponding time scale is then about 3 years. There is an uncertainty in this, as the final jet direction may already be established before the actual merger occurs [58], but the actual merger will surely re-energize the jet, since the jet gets a new foot structure, switching from the dominant compact object of a pair to a single merged compact object. Another uncertainty is the relativistic geometry effects, like boosting, ignored here; relativistic effects could shorten the lag time-scale. The interaction of the newly energized population of hadronic particles then produces both HE photons and neutrinos.

Apart from AGN, for example the interaction of a freely expanding SNIIn-ejecta with the circumstellar medium gives rise to a shock-wave propagating in the dense SN environment, which may accelerate protons to $\mathrm{PeV}$ energies. The cumulative neutrino emission from such process is found to be $\sim 10$ per-cent of the observed IceCube neutrino flux above $60 \mathrm{TeV}$ [60].

The merger of a neutron star binary may result in the formation of a millisecond magnetar remnant surrounded by a low-mass ejecta shell. A portion of the magnetar's rotational energy is deposited behind the ejecta in a pulsar wind nebula. Ions in this pulsar wind may also be accelerated to ultra-high energies, providing HE cosmic rays and neutrinos. The predicted neutrino spectrum from such sources peaks just below the $90 \%$ confidence level sensitivity curve of IceCube Observatory [61]. In optimistic scenarios the cumulative neutrino background resulting from these events may be observed by the IceCube in the near future, in less optimistic scenarios the diffuse flux is potentially within the reach of next-generation neutrino telescopes [61].

\section{Observation of Possible Sources of Low-Frequency GWs}

At present, radio interferometry, notably the technique of very long baseline interferometry (VLBI) provides the finest angular resolution images of AGN jets. The periodicities observed in the parsec-scale jets are consistent with SMBH binaries in the inspiral evolution stage (e.g., [16,17,62]). These sources often exhibit significant periodic variability in their optical brightness or periodic modulation of the shape of their emission lines due to the orbital motion (e.g., [63-66]). In this stage gravitational radiation plays the leading role in the dissipation of the total energy and the reduction of the separation of the binary, surpassing the effect of dynamical friction [67]. The gravitational lifetime of such systems is million to billion years and the GW signal of their merger is considerable only after 
a long time-scale, hence yet undetectable by LISA. This begs the question of how can even tighter binaries with decade-long gravitational lifetime be identified as sources for LISA. Observation of such sources in the electromagnetic spectrum or via HE neutrinos or UHECRs is important for breaking degeneracies in their parameter estimation.

LISA will be sensitive in a frequency range from $0.1 \mathrm{mHz}$ to $1 \mathrm{~Hz}$, being able to detect GWs emmited by massive binaries with total mass $m\left(=m_{1}+m_{2}\right) \in\left[10^{4} \div 10^{7}\right] M_{\text {Sun }}$, mass ratio $v\left(=m_{2} / m_{1}\right) \in[1 / 10 \div 1]$ [9]. The equation of motion of the binary is (e.g., [68]):

$$
\frac{d^{2} \mathbf{r}}{d t^{2}}=-\frac{G m \mathbf{r}}{r^{3}}\left(1+\mathcal{O}(\varepsilon)+\mathcal{O}\left(\varepsilon^{1.5}\right)+\mathcal{O}\left(\varepsilon^{2}\right)+\mathcal{O}\left(\varepsilon^{2.5}\right)+\ldots\right),
$$

where $\mathbf{r}$ is the binary separation $(r=|\mathbf{r}|), \varepsilon=G m / c^{2} / r$ is the post-Newtonian (PN) parameter, employed as expansion paramter, with $G$ the gravitational constant, $c$ the speed of the light and $\mathcal{O}\left(\varepsilon^{n}\right)$ represents the $n$th PN order. Since the gravitational radiation tends to circularize the orbits [69], we discuss circular orbits. In that case the orbital period is given by

$$
T=2 \pi \frac{G m}{c^{3}} \varepsilon^{-3 / 2}
$$

With the gravitational radiation the dominant dissipative effect the binary radiates its orbital angular momentum $L$ at rate $\dot{L}$, from which the inverse of its gravitational lifetime emerges as [58]:

$$
\frac{1}{T_{\mathrm{GW}}}=-\frac{\dot{L}}{L}=\frac{32 c^{3}}{5 G m} \varepsilon^{4} \eta,
$$

where $\eta=m_{1} m_{2} / m^{2}=v /(1+v)^{2}$ is the symmetric mass ratio. The frequency of the GW to leading order is given by:

$$
f_{G W}=\frac{c^{3}}{\pi G m} \varepsilon^{3 / 2}
$$

which is twice the orbital frequency $f_{T}=1 / T$ ( $T$ is the orbital period). We plot in Figure 1 the gravitational lifetime $T_{\mathrm{GW}}$ as function of the total mass $m \in\left[10^{4} M_{\text {Sun }} \div 10^{7} M_{\text {Sun }}\right]$, and orbital period $T \in\left[10^{3} s \div 10^{5} \mathrm{~s}\right]$ (or $T \in[0.27 \mathrm{hr} \div 27.7 \mathrm{hr}]$ ) for three choices of mass ratio $v=1 / 3,1 / 10,1 / 30$. The PN-parameter $\varepsilon$ was expressed with the total mass and the orbital period using Equation (2), and the resulted function $\varepsilon(m, T)$ was substituted to Equation (3). Sub-parsec separated binary systems coalescing presumably on time-scale of up to several decades are the potential sources of the lfGWs: these are either binaries at the high-mass end of the LISA range with up to several days orbital period, or its low-mass end with several hour orbital period (see Figure 1).
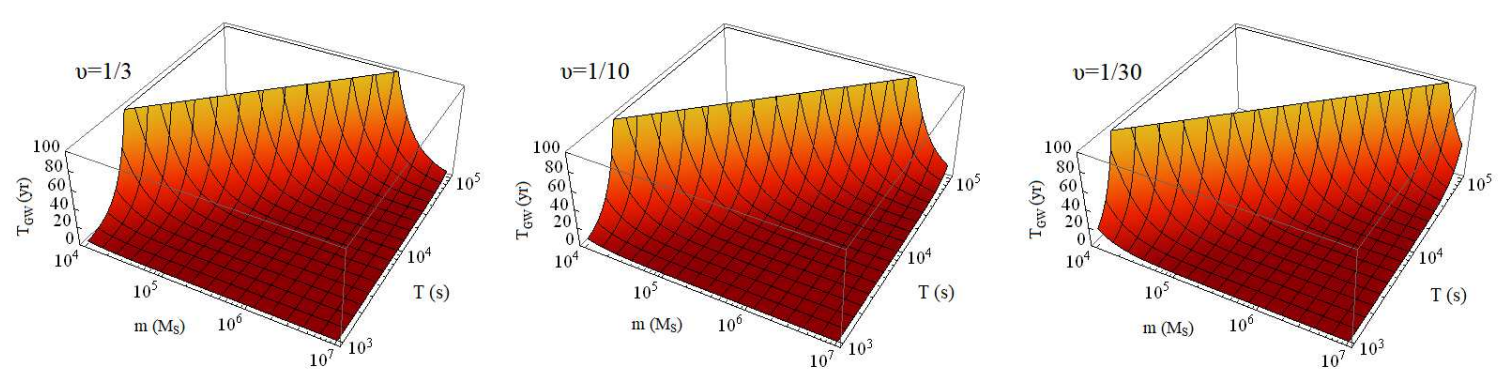

Figure 1. The gravitational lifetime of a binary $T_{\mathrm{GW}}$ as function of the total mass $m$ and orbital period $T$, for choices of mass ratio $v=1 / 3$ (left-sided panel), $v=1 / 10$ (middle panel), $v=1 / 30$ (right-sided panel). 
Such close SMBH systems are impossible to resolve due to their closeness, even with the astronomical techniques giving the finest angular resolutions. Indirect methods, based on the studies of $\mathrm{X}$-shaped radio galaxies, accretion disks with central cavities, ripples in $\mathrm{FeK} \alpha \mathrm{X}$-ray lines, HE neutrino emission are rather explored.

A theoretically promising technique relies on the investigation of the FeK $\alpha$ line. The inner edge of the accretion disk is hot enough to excite this emission line $(E=6.4 \mathrm{keV}, \lambda=0.19 \mathrm{~nm})$. The orbital motion of the secondary BH opens a gap in the disk, and it affects the line profile. The authors of [70] have simulated the FeK $\alpha$ line of the accretion disk of a SMBH in a 10, 20, 50, 90 Schwarzschild radii separated binary (with 10 per-cent error on the separation), and their simulations resulted ripples on the theoretical line-shape induced by such orbital motion, which do not appear on the unperturbed iron line. Considering the closeness of these systems they could be possible sources of strong GW bursts over human lifetime. However, the X-ray observations are far more difficult in technique, than e.g., optical observations, and the $\mathrm{S} / \mathrm{N}$ of the present $\mathrm{X}$-ray spectra does not allow to fit such rippled FeK $\alpha$ models. Additionally, the inner edge of the accretion disk may depend not only on the SMBH mass and spin, but also on the size of the jet-launching region or on the perturbations induced by an accompanying SMBH. Hence the plasma temperature at the modified inner edge cannot reach the level necessary to excite the FeK $\alpha$ emission line.

An important question arises then, on which other ways could we identify such merging binaries. For this purpose particle physical processes following the final coalescence of SMBH binary systems can prove useful.

\section{Detection of Neutrinos by the IceCube Neutrino Observatory}

IceCube, located at the Amundsen-Scott South Pole Station, uses one cubic kilometre of the Antarctic ice as detector matter. The detector strings penetrate up to $2500 \mathrm{~m}$ deep underground, and are sensitive to the Cherenkov radiation of electrons and muons emerging from the interaction of electron and muon neutrinos with the ice. Muons leave track-type paths, such that their origin can be located in the sky within about 1 degree circle. Electrons leave shower-type paths, the directional uncertainty of their origin being several ten degrees. Background events (99.9\% of the events) producing first light in the veto region of IceCube are discarded as entering tracks (usually from cosmic ray muons entering the detector). The deposited energy is also a veto, only the $\mathrm{HE}(\mathrm{TeV}, \mathrm{PeV})$ neutrinos are considered to be extragalactic. Up to date the IceCube Collaboration has published the parameters of 55 cosmic HE neutrino detections [71-73], from which 15 events were track-type. An excellent review of the high-energy neutrino astrophysics with IceCube can be found in [74].

Identification of the origin of these HE neutrinos [71-73] is an actively pursued topic in astroparticle physics consisting of constructing models for the neutrino emission, then comparing their predictions with actual detections. In statistical sense there are two fundamental ways to adjust a model to data. First, determine the best-fitting parameters. Second, answer how likely is the model with a set of parameters, given the data. This method relies on the Fisher concept of likelihood of a set of parameters having an assigned set of values being proportional to the probability, that with them the totality of observations emerge.

The energy spectrum of the UHECRs is measured to be power-law (e.g., [75]). It is assumed that the neutrino spectrum essentially traces the proton spectrum (e.g., [49]), and the source of neutrinos is assumed to emit them according to a power-law energy spectrum. The question is then what is the event rate for a neutrino model predicting a flux given the energy, solid angle and observing frequency. Then a hypothesis test follows, where the null-hypothesis $\left(H_{0}\right)$ is that the data consists solely of background atmospheric neutrino events, and the alternative hypothesis $\left(H_{S}\right)$ states that the data consists of atmospheric neutrino events as well as astrophysical neutrino events produced by a source with some strength and energy spectrum [76]. These latter are outputs of a quantitative model leading to the neutrino emission. The likelihood of obtaining the data given each hypothesis is calculable; the usually employed test statistic is the $\log$ of the likelihood ratio $\lambda$ (e.g., [76]): 


$$
\lambda=-2 \log \frac{P\left(\text { Data } \mid H_{0}\right)}{P\left(\text { Data } \mid H_{S}\right)} .
$$

Large (small) values of $\lambda$ indicate that data is less (rather) compatible with the background hypothesis $H_{0}$. The probability density functions (PDF) $P\left(\right.$ Data $\left.\mid H_{0}\right)$ and $P\left(\right.$ Data $\left.\mid H_{S}\right)$ are calculated using knowledge of the spatial and energy distribution of background and astrophysical neutrino events [76]. The source PDF is a Gaussian, and it depends on the space angle difference between the direction of the reconstructed event $\left(\vec{x}_{i}\right)$ and the source $\left(\vec{x}_{s}\right)$, the reconstruction error estimate $(\sigma)$, and the probability distribution function of the neutrino spectrum given its spectral index $\gamma\left(P\left(E_{i} \mid \gamma\right)\right)$ :

$$
\mathcal{S}\left(\vec{x}_{i}, \vec{x}_{s}, E_{i}, \gamma\right)=\frac{1}{2 \pi \sigma^{2}} \exp \left[-\frac{\left|\vec{x}_{i}-\vec{x}_{S}\right|^{2}}{2 \sigma^{2}}\right] P\left(E_{i} \mid \gamma\right),
$$

where there are tabulated values for $P\left(E_{i} \mid \gamma\right)$ (e.g., [76]). The background PDF depends on the probability of the atmospheric neutrino flux $\left(\phi_{a t m}\right)$ given the energy $\left(E_{i}\right)$, over the solid angle $(\Omega)$ :

$$
\mathcal{B}_{i}=\frac{P\left(E_{i} \mid \phi_{a t m}\right)}{\Omega},
$$

where $P\left(E_{i} \mid \phi_{a t m}\right)$ is precalculated assuming $\phi_{a t m}$ [77]. Then the source and background PDFs are combined, and the likelihood $\mathcal{L}\left(\vec{x}_{s}, n_{s}, \gamma\right)$ is evaluated over all events in the band for a source supposed to emit neutrinos:

$$
\mathcal{L}\left(\vec{x}_{s}, n_{s}, \gamma\right)=\prod_{N}\left[\frac{n_{s}}{N} \mathcal{S}+\left(1-\frac{n_{s}}{N}\right) \mathcal{B}_{i}\right] .
$$

Then the test statistics $\lambda$ written in terms of $\mathcal{L}$ becomes [76]

$$
\lambda=-2 \operatorname{sign}\left(\hat{n}_{s}\right) \log \left[\frac{\mathcal{L}\left(\vec{x}_{s}, 0\right)}{\mathcal{L}\left(\vec{x}_{s}, \hat{n}_{s}, \hat{\gamma}\right)}\right]
$$

The fraction of signal events $\left(n_{s}\right)$ as well as the source spectral index $(\gamma)$ are unknown and must be determined by maximizing the likelihood $\mathcal{L}$. This is done by minimizing the quantity $-\log \mathcal{L}$ with respect to the unknown quantities $n_{s}$ and $\gamma$, obtaining the best value of each of these parameters $\hat{n}_{s}$ and $\hat{\gamma}$.

\section{Discussion: Reorienting Jets, as Sources of High-Energy Neutrinos}

If the spins are misaligned with the orbital angular momentum, a spin-orbit type precession will cause the spin vectors to slowly rotate about the orbital angular momentum vector in the inspiral phase $[78,79]$. For the typical mass ratio of the merging SMBHs $v=m_{2} / m_{1} \in[1 / 30 \div 1 / 3](v \leq 1)$ the spin of the primary SMBH is dominant [58], and the spin of the secondary SMBH can be neglected.

In binary SMBHs with the typical mass ratio the dominant spin-flips, and the jet is reoriented along the spin-flipped dominant spin. This process is the most probable driving mechanism for the formation of the X-shaped radio galaxies [80]. In them the remnant of the old jet appears together with the freshly made jet, forming an X-shape. The old jet-pair has steep continuum spectrum emanated from slowly moving charged particles. The jet-pair formed after the spin-flip is bright and has flat continuum spectrum due to the energetic and fast, charged particles. This morphology can reveal either a recent merger of two SMBHs or the presence of a second active SMBH in the nucleus.

In [51] the blazar PKS 0723-008 was identified as an excellent candidate source of a track-type HE neutrino event, as its spectrum is flat up to high Planck frequencies, and it produced a fivefold-increased radio flux density through the last decade, supporting the scenario of the track-type HE neutrino event related to a binary SMBH merger, more specifically to the plunge of a newly formed jet into the environment of the binary SMBH. The observable consequences are: 
(i) the high Lorentz factor of the freshly made jet due to the gravitational shock-wave,

(ii) enhanced radiation in all EM frequencies,

(iii) the spectrum of the AGN is flat up to $\mathrm{THz}$ frequencies due to the energetic synchrotron-radiating electrons,

(iv) its radio flux density is increasing as the electrons are speeding, leading to enhanced synchrotron radiation,

(v) neutrino emission.

These were all verified to hold true. The sources of neutrinos might be energetic proton-proton collisions, in which the proton energy is larger than the limit to create pions in the process (larger then $290 \mathrm{MeV}$, assuming two protons moving with equal and opposite velocities). The pion decays into a muon and a muon neutrino, while the muon decays into an electron, an anti-muon neutrino and an electron neutrino. If the kinetical energy of the colliding protons cannot supply for the rest-mass of the pion, they fuse into a diproton, which $\beta^{+}$decays into a deuterium, while emitting a positron, an electron-neutrino, and a $\gamma$-ray photon. Therefore the detection of the muon neutrinos has to be explained with HE proton collisions.

Finally we pinpoint here the usefulness of constructing a quantitative model leading to the emission of HE neutrinos, which would give the probability of their detections from a given source. By fitting related parameters of the model (neutrino event fraction rate, spectral index) to already existing neutrino and AGN data, such as the arrival direction and energy distribution of HE neutrinos, position and energy spectrum of AGNs, one could predict how likely an AGN, given its spectral properties, will emit a neutrino at a particular time due to a post-merger SMBH binary at its centre.

Acknowledgments: E.K. and L.Á.G. acknowledge the support of the Hungarian National Research, Development and Innovation Office (NKFI) in the form of the grant 123996. The project has been supported by the European Union, co-financed by the European Social Fund. EFOP-3.6.2-16-2017-00005.

Author Contributions: All authors contributed equally to this work.

Conflicts of Interest: The authors declare no conflict of interest.

\section{References}

1. Cowan, C.L.; Reines, F.; Harrison, F.B.; Kruse, H.W.; McGuire, A.D. Detection of the Free Neutrino: A Confirmation. Science 1956, 124, 103-104.

2. Linsley, J. Eidence for a Primary Cosmic-Ray Particle with Energy $10^{20}$ eV. Phys. Rev. Lett. 1963, 10, $146-148$.

3. Abbott, B.P.; Abbott, R.; Abbott, T.D.; Abernathy, M.R.; Acernese, F.; Ackley, K.; Adams, C.; Adams, T.; Addesso, P.; Adhikari, R.X.; et al. Observation of Gravitational Waves from a Binary Black Hole Merger. Phys. Rev. Lett. 2016, 116, 061102 .

4. Abbott, B.P.; Abbott, R.; Abbott, T.D.; Abernathy, M.R.; Acernese, F.; Ackley, K.; Adams, C.; Adams, T.; Addesso, P.; Adhikari, R.X.; et al. GW151226: Observation of Gravitational Waves from a 22-Solar-Mass Binary Black Hole Coalescence. Phys. Rev. Lett. 2016, 116, 241103.

5. Abbott, B.P.; Abbott, R.; Abbott, T.D.; Acernese, F.; Ackley, K.; Adams, C.; Adams, T.; Addesso, P.; Adhikari, R.X.; Adya, V.B.; et al. GW170104: Observation of a 50-Solar-Mass Binary Black Hole Coalescence at Redshift 0.2. Phys. Rev. Lett. 2017, 118, 221101.

6. Abbott, B.P.; Abbott, R.; Abbott, T.D.; Abernathy, M.R.; Acernese, F.; Ackley, K.; Adams, C.; Adams, T.; Addesso, P.; Adhikari, R.X.; et al. Binary Black Hole Mergers in the First Advanced LIGO Observing Run. Phys. Rev. X 2016, 6, 041015.

7. Abbott, B.P.; Abbott, R.; Abbott, T.D.; Acernese, F.; Ackley, K.; Adams, C.; Adams, T.; Addesso, P.; Adhikari, R.X.; Adya, V.B.; et al. GW170814: A Three-Detector Observation of Gravitational Waves from a Binary Black Hole Coalescence. Phys. Rev. Lett. 2017, 119, 141101.

8. Abbott, B.P.; Abbott, R.; Abbott, T.D.; Acernese, F.; Ackley, K.; Adams, C.; Adams, T.; Addesso, P.; Adhikari, R.X.; Adya, V.B.; et al. GW170817: Observation of Gravitational Waves from a Binary Neutron Star Inspiral. Phys. Rev. Lett. 2017, 119, 161101.

9. Amaro-Seoane, P.; Aoudia, S.; Babak, S.; Binétruy, P.; Berti, E.; Bohé, A.; Caprini, C.; Colpi, M.; Cornish, N.J.; Danzmann, K.; et al. eLISA: Astrophysics and cosmology in the millihertz regime. Gravit. Waves Notes 2013, $6,4-110$. 
10. Begelman, M.C.; Blandford, R.D.; Rees, M.J. Theory of extragalactic radio sources. Rev. Mod. Phys. 1984, 56, 255-351.

11. Villata, M.; Raiteri, C.M. Helical jets in blazars. I. The case of MKN 501. Astron. Astrophys. 1999, 347, $30-36$.

12. Roos, N.; Kaastra, J.S.; Hummel, C.A. A massive binary black hole in 1928+738? Astrophys. J. 1993, 409, 130-133.

13. Britzen, S.; Roland, J.; Laskar, J.; Kokkotas, K.; Campbell, R.M.; Witzel, A. On the origin of compact radio sources. The binary black hole model applied to the gamma-bright quasar PKS 0420-014. Astron. Astrophys. 2001, 374, 784-799.

14. Britzen, S.; Kudryavtseva, N.A.; Witzel, A.; Campbell, R.M.; Ros, E.; Karouzos, M.; Mehta, A.; Aller, M.F.; Aller, H.D.; Beckert, T.; et al. The kinematics in the pc-scale jets of AGN. The case of S5 1803+784. Astron. Astrophys. 2010, 511, A57.

15. Britzen, S.; Zamaninasab, M.; Aller, M.; Aller, H.; Kurtanidze, O.; Vercellone, S.; Richter, G.M.; Witzel, A.; Krichbaum, T.P.; Zensus, J.A. Detecting supermassive binary black holes with VLBI-Discovery of a ring-structure in 3C454.3. J. Phys. Conf. Ser. 2012, 372, 012029.

16. Kun, E.; Gabányi, K.É.; Karouzos, M.; Britzen, S.; Gergely, L.Á. A spinning supermassive black hole binary model consistent with VLBI observations of the S5 1928+738 jet. Mon. Not. R. Astron. Soc. 2014, 445, 1370-1382.

17. Kun, E.; Frey, S.; Gabányi, K.É.; Britzen, S.; Cseh, D.; Gergely, L.Á. Constraining the parameters of the putative supermassive binary black hole in PG 1302-102 from its radio structure. Mon. Not. R. Astron. Soc. 2015, 454, 1290-1296.

18. Bon, E.; Jovanović, P.; Marziani, P.; Shapovalova, A.I.; Bon, N.; Jovanović, V.B.; Borka, D.; Sulentic, J.; Popović, L.Č. The First Spectroscopically Resolved Sub-parsec Orbit of a Supermassive Binary Black Hole. Astrophys. J. 2012, 759, 118-126.

19. Keel, W.C.; Lintott, C.J.; Maksym, W.P.; Bennert, V.N.; Chojnowski, S.D.; Moiseev, A.; Smirnova, A.; Schawinski, K.; Sartori, L.F.; Urry, C.M.; et al. Fading AGN Candidates: AGN Histories and Outflow Signatures. Astrophys. J. 2017, 835, 256.

20. Searle, L.; Zinn, R. Compositions of halo clusters and the formation of the galactic halo. Astrophys. J. 1978, 225, 357-379.

21. Kauffmann, G.; Haehnelt, M. A unified model for the evolution of galaxies and quasars. Mon. Not. R. Astron. Soc. 2000, 311, 576-588.

22. Andrade-Santos, F.; Bogdán, Á.; Romani, R.W.; Forman, W.R.; Jones, C.; Murray, S.S.; Taylor, G.B.; Zavala, R.T. Binary Black Holes, Gas Sloshing, and Cold Fronts in the X-ray Halo Hosting 4C+37.11. Astrophys. J. 2016, $826,91$.

23. Komossa, S. Observational evidence for binary black holes and active double nuclei. Mem. Soc. Astron. Ital. 2006, 77, 733-741.

24. Begelman, M.C.; Blandford, R.D.; Rees, M.J. Massive black hole binaries in active galactic nuclei. Nature 1980, 287, 307-309.

25. Merritt, D.; Milosavljevic, M. Massive Black Hole Binary Evolution. Living Rev. Relativ. 2005, 8, 8.

26. Mestel, L. On the galactic law of rotation. Mon. Not. R. Astron. Soc. 1963, 126, 553-575.

27. Hobson, M.P.; Efstathiou, G.P.; Lasenby, A.N. General Relativity: An Introduction for Physicists; Cambridge University Press: Cambridge, UK, 2006; ISBN-13: 978-0-521-82951-9.

28. Beckmann, V.; Shrader, C. Active Galactic Nuclei; Wiley-VCH: Weinheim, Germany, 2012; ISBN 978-3-527-41078-1.

29. Tchekhovskoy, A.; McKinney, J.C.; Narayan, R. General Relativistic Modeling of Magnetized Jets from Accreting Black Holes. J. Phys. Conf. Ser. 2012, 372, 012040.

30. Boettcher, M.; Harris, D.E.; Krawczynski, H. (Eds.) Relativistic Jets from Active Galactic Nuclei; Wiley-VCH: Weinheim, Germany, 2012; ISBN 978-3-527-41037-8.

31. Blandford, R.D.; Znajek, R.L. Electromagnetic extraction of energy from Kerr black holes. Mon. Not. R. Astron. Soc. 1977, 179, 433-456.

32. Blandford, R.D.; Payne, D.G. Hydromagnetic flows from accretion discs and the production of radio jets. Mon. Not. R. Astron. Soc. 1982, 199, 883-903.

33. Britzen, S.; Fendt, C.; Eckart, A.; Karas, V. A new view on the M 87 jet origin: Turbulent loading leading to large-scale episodic wiggling. Astron. Astrophys. 2017, 601, A52.

34. Rybicki, G.B.; Lightman, A.P. Radiative Processes in Astrophysics; Wiley-VCH: Weinheim, Germany, 1985; ISBN 978-0-471-82759-7. 
35. Klein, U.; Fletcher, A. Galactic and Intergalactic Magnetic Fields; Springer International Publishing: Cham, Switzerland, 2015; ISBN 978-3-319-08941-6.

36. Kellermann, K.I.; Sramek, R.; Schmidt, M.; Shaffer, D.B.; Green, R. VLA observations of objects in the Palomar Bright Quasar Survey. Astron. J. 1989, 98, 1195-1207.

37. Padovani, P. The Radio Loud Fraction of QSOS and its Dependence on Magnitude and Redshift. Mon. Not. R. Astron. Soc. 1993, 263, 461-470.

38. Pierre Auger Collaboration. Correlation of the Highest-Energy Cosmic Rays with Nearby Extragalactic Objects. Science 2007, 318, 938-943.

39. Abraham, J.; Abreu, P.; Aglietta, M.; Aguirre, C.; Allard, D.; Allekotte, I.; Allen, J.; Allison, P.; Alvarez-Muñiz, J.; Ambrosio, M.; et al. Auger Collaboration. Correlation of the highest-energy cosmic rays with the positions of nearby active galactic nuclei. Astropart. Phys. 2008, 29, 188-204.

40. Saba, I.; Becker Tjus, J.; Halzen, F. Limits on the source properties of FR-I galaxies from high-energy neutrino and gamma observations. Astropart. Phys. 2013, 48, 30-36.

41. Kalashev, O.; Semikoz, D.; Tkachev, I. Neutrinos in IceCube from active galactic nuclei. J. Exp. Theor. Phys. 2015, 120, 541-548.

42. Aartsen, M.G.; Abraham, K.; Ackermann, M.; Adams, J.; Aguilar, J.A.; Ahlers, M.; Ahrens, M.; Altmann, D.; Andeen, K.; Anderson, T.; et al. IceCube Collaboration The Contribution of Fermi-2LAC Blazars to Diffuse TeV-PeV Neutrino Flux. Astrophys. J. 2017, 835, 45.

43. Mannheim, K.; Biermann, P.L. Photomeson production in active galactic nuclei. Astron. Astrophys. 1989, 221, 211-220.

44. Begelman, M.C.; Rudak, B.; Sikora, M. Consequences of relativistic proton injection in active galactic nuclei. Astrophys. J. 1990, 362, 38.

45. Mücke, A.; Rachen, J.P.; Engel, R.; Protheroe, R.J.; Stanev, T. Photohadronic Processes in Astrophysical Environments. Publ. Astron. Soc. Aust. 1999, 16, 160-166.

46. Biermann, P.L.; Strittmatter, P.A. Synchrotron emission from shock waves in active galactic nuclei. Astrophys. J. 1987, 322, 643-649.

47. Stecker, F.W.; Done, C.; Salamon, M.H.; Sommers, P. High-energy neutrinos from active galactic nuclei. Phys. Rev. Lett. 1991, 66, 2697-2700.

48. Mannheim, K. High-energy neutrinos from extragalactic jets. Astropart. Phys. 1995, 3, 295-302.

49. Becker, J.K.; Biermann, P.L. Neutrinos from active black holes, sources of ultra high energy cosmic rays. Astropart. Phys. 2009, 31, 138-148.

50. Kun, E.; Wiita, P.J.; Gergely, L.Á.; Keresztes, Z.; Gopal-Krishna; Biermann, P.L. Constraints on supermassive black hole spins from observations of active galaxy jets. Astron. Nachr. 2013, 334, 1024-1027.

51. Kun, E.; Biermann, P.L.; Gergely, L.Á. A flat-spectrum candidate for a track-type high-energy neutrino emission event, the case of blazar PKS 0723-008. Mon. Not. R. Astron. Soc. Lett. 2017, 466, L34-L38.

52. Biermann, P.L.; Caramete, L.I.; Fraschetti, F.; Gergely, L.A.; Harms, B.C.; Kun, E.; Lundquist, J.P.; Meli, A.; Nath, B.B.; Seo, E.S.; et al. The Nature and Origin of Ultra-High Energy Cosmic Ray Particles. arXiv 2016, arXiv:1610.00944.

53. Abbott, B.P.; Abbott, R.; Adhikari, R.X.; Ananyeva, A.; Anderson, S.B.; Appert, S.; Arai, K.; Araya, M.C.; Barayoga, J.C.; Barish, B.C.; et al. Multi-messenger Observations of a Binary Neutron Star Merger. Astrophys. J. Lett. 2017, 848, L12.

54. Falcke, H.; Biermann, P.L. The jet-disk symbiosis. I. Radio to X-ray emission models for quasars. Astron. Astrophys. 1995, 293, 665-682.

55. Markoff, S.; Falcke, H.; Yuan, F.; Biermann, P.L. The Nature of the 10 kilosecond X-ray flare in Sgr A*. Astron. Astrophys. 2001, 379, L13-L16.

56. Blandford, R.D.; Königl, A. Relativistic jets as compact radio sources. Astrophys. J. 1979, 232, $34-48$.

57. Marscher, A.P.; Jorstad, S.G.; D’Arcangelo, F.D.; Smith, P.S.; Williams, G.G.; Larionov, V.M.; Oh, H.; Olmstead, A.R.; Aller, M.F.; Aller, H.D.; et al. The inner jet of an active galactic nucleus as revealed by a radio-to- $\gamma$-ray outburst. Nature 2008, 452, 966-969.

58. Gergely, L.Á.; Biermann, P.L. The Spin-Flip Phenomenon in Supermassive Black hole binary mergers. Astrophys. J. 2009, 697, 1621. 
59. Abbott, B.P.; Abbott, R.; Abbott, T.D.; Acernese, F.; Ackley, K.; Adams, C.; Adams, T.; Addesso, P.; Adhikari, R.X.; Adya, V.B.; et al. Gravitational Waves and Gamma-Rays from a Binary Neutron Star Merger: GW170817 and GRB 170817A. Astrophys. J. 2017, 848, L13.

60. Petropoulou, M.; Coenders, S.; Vasilopoulos, G.; Kamble, A.; Sironi, L. Pint-source and diffuse high-energy neutrino emission from Type IIn supernovae. Mon. Not. R. Astron. Soc. 2017, 470, 1881-1893.

61. Fang, K.; Metzger, B.D. High-energy Neutrinos from Millisecond Magnetars Formed from the Merger of Binary Neutron Stars. Astrophys. J. 2017, 849, 153.

62. Britzen, S.; Qian, S.J.; Steffen, W.; Kun, E.; Karouzos, M.; Gergely, L.; Schmidt, J.; Aller, M.; Aller, H.; Krause, M.; et al. A swirling jet in the quasar 1308+326. Astron. Astrophys. 2017, 602, A29.

63. Graham, M.J.; Djorgovski, S.G.; Stern, D.; Drake, A.J.; Mahabal, A.A.; Donalek, C.; Glikman, E.; Larson, S.; Christensen, E. A systematic search for close supermassive black hole binaries in the Catalina Real-time Transient Survey. Mon. Not. R. Astron. Soc. 2015, 453, 1562-1576.

64. Charisi, M.; Bartos, I.; Haiman, Z.; Price-Whelan, A.M.; Graham, M.J.; Bellm, E.C.; Laher, R.R.; Márka, S. A population of short-period variable quasars from PTF as supermassive black hole binary candidates. Mon. Not. R. Astron. Soc. 2016, 463, 2145-2171.

65. Bon, E.; Zucker, S.; Netzer, H.; Marziani, P.; Bon, N.; Jovanović, P.; Shapovalova, A.I.; Komossa, S.; Gaskell, C.M.; Popović, L.Č.; et al. Evidence for Periodicity in 43 year-long Monitoring of NGC 5548. Astrophys. J. Suppl. Ser. 2016, 225, 29.

66. Bon, E.; Marziani, P.; Bon, N. Periodic optical variability of AGN. Proc. Int. Astron. Union 2017, 324, $176-179$.

67. Binney, J.; Tremaine, S. Galactic Dynamics; Princeton University Press: Princeton, NJ, USA, 1987; ISBN 9781400828722.

68. Kidder, L.E. Coalescing binary systems of compact objects to (post) ${ }^{5 / 2}$-Newtonian order. V. Spin Effects. Phys. Rev. D 1995, 52, 821-847.

69. Lincoln, C.W.; Will, C.M. Coalescing binary systems of compact objects to (post) ${ }^{5 / 2}$-Newtonian order: Late-time evolution and gravitational-radiation emission. Phys. Rev. D 1990, 42, 1123-1143.

70. McKernan, B.; Ford, K.E.S.; Kocsis, B.; Haiman, Z. Ripple effects and oscillations in the broad FeK $\alpha$ line as a probe of massive black hole mergers. Mon. Not. R. Astron. Soc. 2013, 432, 1468-1482.

71. IceCube Collaboration. Observation of High-Energy Astrophysical Neutrinos in Three Years of IceCube Data. Phys. Rev. Lett. 2014, 113, 101101.

72. IceCube Collaboration. The IceCube Neutrino Observatory-Contributions to ICRC 2015 Part II: Atmospheric and Astrophysical Diffuse Neutrino Searches of All Flavors. arXiv 2015, arXiv:1510.05223.

73. Schoenen, S.; Raedel, L. Detection of a Multi-PeV Neutrino-Induced Muon Event from the Northern Sky with IceCube. Available online: http://www.astronomerstelegram.org/?read=7856 (accessed on 24 January 2018).

74. Halzen, F. High-energy neutrino astrophysics. Nat. Phys. 2017, 13, 232-238.

75. IceCube Collaboration. Measurement of the cosmic ray energy spectrum with IceTop-73. Phys. Rev. D 2013, 88,042004 .

76. Braun, J.; Dumm, J.; De Palma, F.; Finley, C.; Karle, A.; Montaruli, T. Methods for point source analysis in high energy neutrino telescopes. Astropart. Phys. 2008, 29, 299-305.

77. Barr, G.D.; Gaisser, T.K.; Lipari, P.; Robbins, S.; Stanev, T. Three-dimensional calculation of atmospheric neutrinos. Phys. Rev. D 2004, 70, 023006.

78. Barker, B.M.; O'Connell, R.F. Gravitational two-body problem with arbitrary masses, spins, and quadrupole moments. Phys. Rev. D 1975, 12, 329-335.

79. Barker, B.M.; O'Connell, R.F. The gravitational interaction: Spin, rotation, and quantum effects-A review. Gen. Relativ. Gravit. 1979, 11, 149-175.

80. Gopal-Krishna; Biermann, P.L.; Gergely, L.Á; Wiita, P.J. On the origin of X-shaped radio galaxies. Res. Astron. Astrophys. 2012, 12, 127-146.

(C) 2018 by the authors. Licensee MDPI, Basel, Switzerland. This article is an open access article distributed under the terms and conditions of the Creative Commons Attribution (CC BY) license (http:/ / creativecommons.org/licenses/by/4.0/). 